OPEN ACCESS

Edited by:

Allen D. Smith,

United States Department of

Agriculture, United States

Reviewed by:

Yixin Lu,

Northeast Agricultural University,

China

Natasa llic,

Institute for the Application of

Nuclear Energy (INEP), Serbia

*Correspondence:

Mingyuan Liu

liumy36@163.com

Xiaolei Liu

liuxlei@163.com

${ }^{+}$These authors have contributed equally to this work

Specialty section: This article was submitted to Microbial Immunology, a section of the journal

Frontiers in Immunology

Received: 21 April 2021 Accepted: 09 June 2021

Published: 23 June 2021

Citation:

Jin X, Bai X, Zhao Y, Dong Z, Pang J, Liu M and Liu X (2021) Nrf2 Participates in M2 Polarization by

Trichinella spiralis to Alleviate

TNBS-Induced Colitis in Mice.

Front. Immunol. 12:698494. doi: 10.3389/fimmu.2021.698494

\section{Nrf2 Participates in M2 Polarization by Trichinella spiralis to Alleviate TNBS-Induced Colitis in Mice}

\author{
Xuemin $\mathrm{Jin}^{1 \dagger}$, Xue $\mathrm{Bai}^{1+}$, Ying Zhao ${ }^{2 \dagger}$, Zijian Dong ${ }^{1}$, Jianda Pang ${ }^{1}$, Mingyuan Liu ${ }^{1,3^{*}}$ \\ and Xiaolei Liu ${ }^{1 *}$ \\ ${ }^{1}$ Key Laboratory of Zoonosis Research, Ministry of Education, Institute of Zoonosis, College of Veterinary Medicine, Jilin \\ University, Changchun, China, ${ }^{2}$ Department of Nephrology, First Hospital of Jilin University, Changchun, China, ${ }^{3}$ Jiangsu \\ Co-Innovation Center for Prevention and Control of Important Animal Infectious Diseases and Zoonoses, Yangzhou, China
}

Trichinella spiralis induced alternative activated macrophages (M2), leading to protect against Crohn's disease, known as Th1 -related inflammation, which enhances oxidative stress in the host. However, the relationship of oxidative stress and T. spiralis -mediated immune response is still unknown. In our study, we showed that nuclear factor erythroid 2-related factor-2 (Nrf2), a key transcription factor in antioxidant, participated in M2 polarization induced by T. spiralis muscle larval excretory/secretory (ES) products in vitro. ES -treated M2 were injected intravenously after TNBS challenge and we demonstrated that ES-M could alleviate the severity of the colitis in mice. Adoptive transfer of ES -treated M2 decreased the level of IFN- $\gamma$ and increased the levels of IL-4 and IL-10 in vivo. However, the capacity of ES -treated Nrf2 KO macrophages to treat colitis was dramatically impaired. ES -treated Nrf2 KO macrophages was insufficient to result in the elevated levels of IL-4 and IL-10. These findings indicate that Nrf2 was required for M2 polarization induced by $T$. spiralis ES to alleviate colitis in mice.

Keywords: Trichinella spiralis, macrophage, TNBS, colitis, Th2, Nrf2

\section{INTRODUCTION}

Crohn's disease $(\mathrm{CD})$ is a chronic relapsing inflammatory condition of the gastrointestinal tract with increased production of Th1 cells (1). Countries where helminth infection are endemic have a lower incidence of $\mathrm{CD}$ than non-endemic countries, and there have been interesting reports of the beneficial effects of helminths in CD patients (2), indicating that the regulatory effect of eliminating helminths often leads to imbalances in the immune system and increases immune-mediated diseases (3). Several studies in animals and clinical trials provide strong evidence that helminth can downregulate CD -specific immune responses (3). Unfortunately, this kind of therapy is hard to accept for patients because of the fear of helminths.

Macrophages play crucial roles in immune responses and are the main target in the treatment of CD. Helminths alleviate colitis through polarization of alternatively activated macrophages (M2) (4), which mediate Th2 type responses, contributing to suppression of Th1 type response (5). Recently, cellular immunotherapy is suggested to be a novel therapeutic option to downregluate colitis -related immune response and inflammation (6-8). Trichinella spiralis muscle larval excretory/secretory (MLES) products induce M2, which can ameliorate inflammation of colitis (9). 
Macrophage-based therapy offers hope for the development of a safe, effective and viable treatment for inflammatory diseases in humans (10). However, the mechanism of helminth -induced macrophages remain unclear.

Despite tremendous efforts, the etiology of CD remains unclear. It has been generally accepted that $\mathrm{CD}$ is related to strong oxidative stress and is an important factor in causing colon inflammation (11). The nuclear factor (erythroid-derived 2)-like-2 factor (Nrf2) is a key regulatory transcription factor in the regulation of antioxidant response element-dependent genes (12). Induction of $\mathrm{Nrf} 2$ pathway decreases uncontrolled inflammation such as colitis (13). It has been reported that activation of Nrf2 leads to M2 macrophage polarization (14, 15). Recent study have demonstrated that upregulated Nrf2 expression contributes to immnunomudulatory role of $T$. spiralis infection in host (16), however, details related to the mechanism is still unknown.

In our study, we aimed to investigate the role of Nrf2 in the development of macrophages polarized by T. spiralis. In vitro, we found that murine bone marrow-derived macrophages were activated to M2 phenotype by ES of T. spiralis in Nrf2 dependent manner. We demonstrated that ES -M2 had a therapeutic effect on 2,4,6-trinitrobenzene sulfonic acid (TNBS)-induced CD model in mice. ES -treated M2 could downregulate the Th1-related inflammation and upregulate the Th2 immune response in vivo. However, the capacity of ES treated Nrf2 KO macrophages to treat colitis was dramatically impaired. Our results provided a new evidence of ES -treated M2, which may lead to a potentially new approach in the treatment of $\mathrm{CD}$ and revealed that Nrf2 participated in the development of ES -treated M2.

\section{MATERIALS AND METHODS}

\section{Animals and Ethics Statement}

$\mathrm{BALB} / \mathrm{c}$ mice (female, 6-8 weeks old) and C57JBL/6 mice (female, 6-8 weeks old), and Wistar rats (female, 6-8 weeks old) were purchased from the Experimental Animal Centre of College of Basic Medical Sciences, Jilin University (Changchun, China). Nrf2 knockout (KO) C57BL/6 mice were purchased from the Jackson Laboratory (Bar Harbor, ME, USA) and kept in a temperature-controlled room $\left(22 \pm 2^{\circ} \mathrm{C}\right)$ under a $12 \mathrm{~h}$ darklight cycle. All animal experiments were performed according to regulations of the Administration of Affairs Concerning Experimental Animals in China. The protocol was approved by the Institutional Animal Care and Use Committee of Jilin University (20170318) (8).

\section{T. spiralis and Preparation of ES}

The T. spiralis isolate (ISS534) was obtained from a naturally infected domestic pig in Henan Province in China. Preparation of muscle larvae ES was obtained as described previously (17). Wistar rats were orally infected with 3000 infective larvae, and $T$. spiralis muscle larvae were recovered at 35 days post infection (dpi). All T. spiralis muscle larvae were washed and incubated separately in prewarmed serum-free RPMI 1640 medium containing $2 \mathrm{mM}$ l-glutamine, $100 \mathrm{U} / \mathrm{ml}$ penicillin, and 100 $\mu \mathrm{g} / \mathrm{ml}$ streptomycin at $37^{\circ} \mathrm{C}$ under $5 \%$ atmospheric $\mathrm{CO}_{2}$ for $24 \mathrm{~h}$. After centrifugation, the supernatant containing ES products was dialyzed and concentrated. According to the manufacturer's instructions, the endotoxin was removed from the protein by using the ToxOut ${ }^{\mathrm{TM}}$ High Capacity Endotoxin Removal kit (Biovision, USA). There was about approximately $0.134 \mathrm{EU} / \mathrm{ml}$ residual endotoxin existing in MLES, approximately equivalent to $20 \mathrm{pg} / \mathrm{mg}$ endotoxin $(17,18)$. The protein concentration was quantified with a BCA protein assay kit (Thermo Scientific). We made three different preparations of ES products from muscle larvae of T. spiralis to perform several independent experiments. One representative experiment is shown here.

\section{Bone Marrow-Derived Macrophages (BMDM) Isolation, Culture, and Stimulation}

Bone marrow-derived macrophages (BMDM) were generated from murine bone marrow cells as described previously (19). Briefly, bone marrow cells were obtained from wild type (WT) and Nrf2 KO C57JBL/6 mice and cultured in DMEM medium containing growth factors of $20 \mathrm{ng} / \mathrm{ml}$ recombinant M-CSF (Sigma-Aldrich) and $10 \% \mathrm{FBS}$ at $37^{\circ} \mathrm{C}, 5 \% \mathrm{CO}_{2}$. The culture medium was replaced every $72 \mathrm{~h}$. Adherent cells were collected after 6 days whereas non-adherent cells were removed by washing the plates with phosphate buffer solution (PBS) twice. Macrophages were enriched by positive selection with anti- F4/ 80 magnetic beads (Miltenyi Biotec). The enriched F4/80 macrophages were typically of $>90 \%$ purity as determined by flow cytometry (Figure 1A). To determine the phenotype of macrophages, macrophages were stimulated with sterile PBS, ES or LPS alone for $24 \mathrm{~h}$ or macrophages were pre-treated with ES $(50 \mu \mathrm{g} / \mathrm{ml})$ for $24 \mathrm{~h}$ before stimulation with LPS $(100 \mathrm{ng} / \mathrm{ml}$, Sigma-Aldrich) for $24 \mathrm{~h}$. Cell culture supernatants were collected and stored at $-80^{\circ} \mathrm{C}$ for the following experiments.

\section{RNA Extraction and Real Time (RT)-PCR}

mRNA expression levels were quantified using RT-PCR as described previously (8). Briefly, RNA from $5 \times 10^{6}$ cells from each sample was extracted with Trizol reagent (Invitrogen) according to the manufacturer's instructions. Complementary DNA (cDNA) was synthesized with a reverse transcriptase kit (Vazyme, Nanjing, China). RT-PCR was performed using SYBR Green QPCR Master Mix (TaKaRa, Japan). The primers used for RT-PCR are listed in Table $\mathbf{1 .}$

\section{Establishment of Colitis Model for Adoptive Transfer of Macrophages in Mice} Colitis was generated by intrarectal administration of 2,4,6trinitrobenzene sulfonic acid (TNBS) solution (sigma, USA) as described previously (8). Briefly, BALB/c mice were fasted for $24 \mathrm{~h}$ with free access to drinking water and then were anesthetized using sodium pentobarbital $(50 \mathrm{mg} / \mathrm{kg}$, ip). Next, a catheter was inserted through the anus to approximately the level of the splenic flexure and the colon was then infused with 


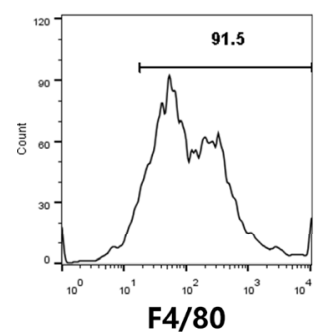

B

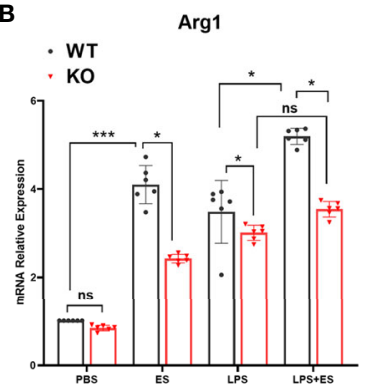

C

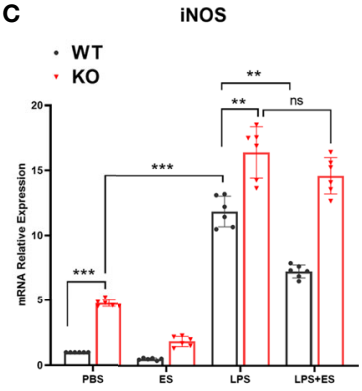

FIGURE 1 | Expression of iNOS and Arg1 in wild type (WT) or Nrf2 KO macrophages induced by T. spiralis ES. Macrophages were enriched by positive selection with anti- F4/80 magnetic beads. The enriched F4/80+ macrophages were typically of $>90 \%$ purity as determined by flow cytometry (A). Total RNA was extracted from cells and mRNA expression levels of $\operatorname{Arg} 1 \mathbf{( B )}$ and iNOS (C) were quantified using RT-PCR. The results are presented as the mean \pm SD of each group ( $\mathrm{n}=6$ ) ${ }^{*} \mathrm{P}<0.05,{ }^{* \star} \mathrm{P}<0.01,{ }^{\star \star *} \mathrm{P}<0.001$, ns, no significance, as indicated by the line (one-way ANOVA with Tukey's post hoc test).

TABLE 1 | The primers of quantitative RT-PCR.

\begin{tabular}{lll}
\hline Genes & \multicolumn{1}{c}{ Primer } & \multicolumn{1}{c}{ Sequence $\left(\mathbf{5}^{\prime} \rightarrow \mathbf{3}^{\prime} \mathbf{)}\right.$} \\
\hline iNos & Forward primer & AAAGTGACCTGAAAGAGGAAAAGGA \\
& Reverse primer & TTGGTGACTCTAGGGTCATCTTGTA \\
Arg-1 & Forward primer & CTCCAAGCCAAAGTCCTTAGAG \\
& Reverse primer & AGGAGCTGTCATTAGGGACATC \\
GAPDH & Forward primer & ACTCCACTCACGGCAAATC \\
& Reverse primer & TCTCCATGGTGGTGAAGACA \\
\end{tabular}

mixture (5\% TNBS and absolute ethyl ethanol with ratio $1: 1$ ) intrarectally. The mice in control group were administrated with $50 \%$ ethyl ethanol in the same way. The mice were allowed to eat and drink ad libitum from $1 \mathrm{~h}$ after the operation. Mice were randomly divided into 4 groups of six mice each. For adoptive transfer, WT and Nrf2 KO macrophages stimulated with ES were washed (x3) with sterile PBS, and $1 \times 10^{6}$ cells in $500 \mu \mathrm{L}$ of sterile PBS were injected intravenously (i.v.) immediately after TNBS challenge. Three days later, mice were humanely euthanized by $\mathrm{CO}_{2}$, and then the colon and spleen were collected for the following experiments. The effect of macrophages on TNBS induced colitis was evaluated in three independent experiments.

\section{Assessment of Colitis}

The mice in each group were observed daily and given a clinical disease score (disease activity index, DAI) ranging from 0 to 12 (Table 2). DAI was assessed by body weight loss, stool consistency, and stool bleeding, which were all recorded everyday as described previously (8). Approximately $1 \mathrm{~cm}$ of colon was resected for histopathology examination, fixed in $4 \%$

TABLE 2 | Disease activity index score parameters (DAl).

\begin{tabular}{lllc}
\hline Weight loss(\%) & \multicolumn{1}{c}{ Stool } & Bloody stool & Index \\
\hline $\mathbf{0 - 1} \%$ & Normal & None & 0 \\
$\mathbf{1 - 4 \%}$ & Soft and shaped & Between & 1 \\
$\mathbf{4 - 8} \%$ & Loose & Slight & 2 \\
$\mathbf{8 - 1 2 \%}$ & Between & Between & 3 \\
$\mathbf{8 1 2} \%$ & Diarrhea & Gross bleeding & 4
\end{tabular}

neutral-buffered formalin, embedded in paraffin, sectioned at $5 \mu \mathrm{m}$ thickness and stained separately with hematoxylin and eosin (H\&E), according to standard protocols as described previously (8). The histological damage scoring was based on the following 2 parameters: epithelial lesion ( 0 , none damage; 1 , some loss of goblet cells; 2 , extensive loss of goblet cells; 3 , some loss of crypts; 4 , extensive loss of crypts); infiltration (0, none infiltration; 1, infiltration around crypt bases; 2, infiltration spreading to muscularis mucosa; 3 , extensive infiltration in the muscularis mucosa with abundant oedema; 4, infiltration spreading to submucosa). The total score ranged from 0 to 8 .

\section{MPO Activity Assay}

Inflammatory cell (polymorphonuclear neutrophil) infiltration into colonic tissue was quantified by measuring MPO activity with an MPO assay kit (Nanjing Jiancheng Bio-engineering Institute, China), following the manufacturer's instructions. MPO activity was expressed as units per gram of total protein (U/g) (20).

\section{Flow Cytometry Staining}

The stimulated macrophages were preincubated with Fc Block (anti-CD16/CD32, BD Biosciences) for $10 \mathrm{~min}$ at $4^{\circ} \mathrm{C}$. These cells were stained with $\mathrm{PE}$-conjugated mAbs to CD11c (Biolegend). Macrophages were fixed, permeabilized using a FIX/PERM set (Biolegend) for $10 \mathrm{~min}$ at room temperature prior to intracellular staining with PE-anti-CD206 (BD Biosciences). Splenocytes were separated from spleens of mice and cultured in the medium above for $36 \mathrm{~h} .2 \mu \mathrm{L} / \mathrm{ml}$ stimulation cocktail (Thermo Invitrogen, USA) was added to the RPMI 1640 medium for the last $10 \mathrm{~h}$, and $0.66 \mu \mathrm{L} / \mathrm{ml}$ Golgistop ${ }^{\text {TM }}$ (BD Biosciences) for the last $5 \mathrm{~h}$. Cells were first preincubated with $\mathrm{Fc}$ Block for $30 \mathrm{~min}$ and stained for PerCP-Cy5.5-anti-CD3 and FITC-anti-CD4 antibodies (BD Biosciences) for $35 \mathrm{~min}$ at $4^{\circ} \mathrm{C}$ in the dark. Then cells were fixed, permeabilized using a FIX/PERM set (Biolegend) for $10 \mathrm{~min}$ at room temperature prior to intracellular staining with PE-anti-IL-4, APC-anti- IFN- $\gamma$ antibodies or PE-anti-IL-10. Samples were analyzed by using a 
BD FACS Calibur flow cytometer and FlowJo software (Tree star Inc, Ashland, OR).

\section{Determination of Colon Cytokines}

Cytokine levels of IFN- $\gamma$, IL- 4 or IL-10 in the colon were determined as described previously (8). Briefly, a segment of the colon was excised and washed twice in clean PBS containing penicillin and streptomycin. Then, the colon was further cut into $1 \mathrm{~cm}^{2}$ pieces and placed in 24-well flat bottom-well culture plates with $1 \mathrm{ml}$ RPMI 1640 supplemented with $100 \mathrm{U} / \mathrm{ml}$ penicillin and $100 \mu \mathrm{g} / \mathrm{ml}$ streptomycin at $37^{\circ} \mathrm{C}, 5 \% \mathrm{CO}_{2}$ for $24 \mathrm{~h}$. The supernatant was collected, and cellular debris was removed by centrifugation. The levels of cytokines (IFN- $\gamma$, IL- 4 or IL-10) in the supernatant were quantified by ELISA.

\section{Statistical Analysis}

All results are expressed as the mean \pm SD. Statistical analysis was performed using the GraphPad Prism 8 software for Windows. One-way, two-way analysis of variance (ANOVA) and independent exponent $t$ test were used to compare the means and determine statistically significant differences between different conditions. $\mathrm{p}$ values are expressed as ${ }^{*} \mathrm{p}<0.05$, ${ }^{* *} \mathrm{p}<0.01$ and ${ }^{* *} \mathrm{p}<0.001$.

\section{RESULTS}

\section{Nrf2 Was Involved in M2 Polarization Induced by T. spiralis ES In Vitro}

To investigate whether Nrf2 is involved in M2 polarization induced by $T$. spiralis ES, we compared the polarization of ES-treated macrophages from wild type (WT) mice and Nrf2 KO mice. Our results showed that ES increased the expression of Arginase-1 (Arg1) in WT macrophages but the enhanced expression of Arg1 and CD206 (M2 markers) induced by ES was decreased in Nrf2 KO macrophages (Figures 1B, 2C, D). We found that ES inhibited LPS -induced high level of iNOS and CD11c (M1 markers) in WT macrophages while there was no significant difference between LPS group and LPS + ES group in Nrf2 KO macrophages (Figures 1C, 2A, B). M1 is characterized by the expression of high levels of proinflammatory cytokines such as IL-12 (21). LPS can stimulate the level of pro-inflammatory cytokine IL-12, which was significantly

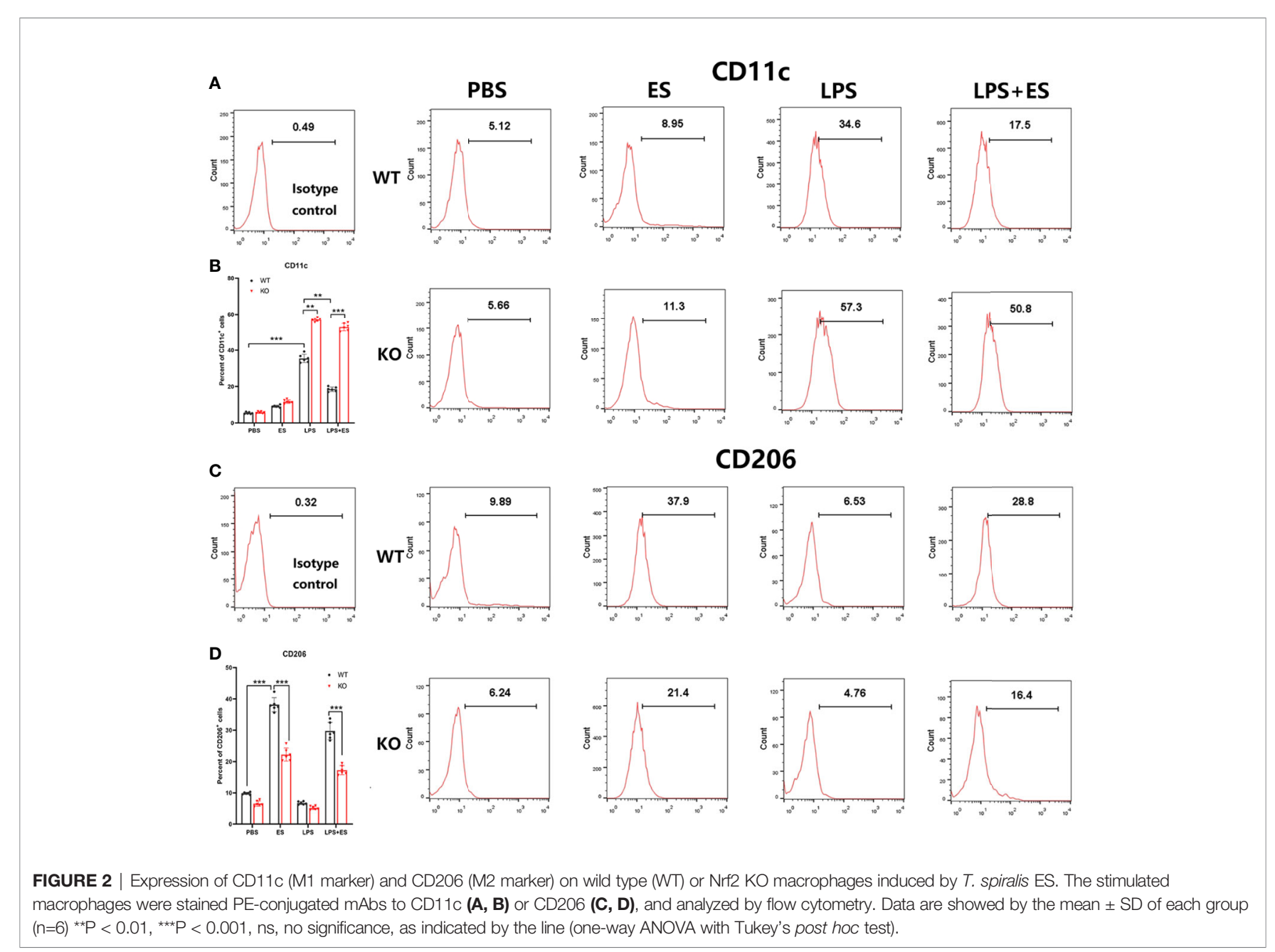


decreased by ES in WT macrophages, but not Nrf2 KO macrophages (Figure 3). In contrast, M2 function in resolving inflammation and is characterized by the expression of antiinflammatory cytokine IL-10 (22). We observed that both ES and LPS enhanced the level of IL-10, and combination of ES and LPS significantly increased IL-10 level in macrophages compared to PBS, ES or LPS group. However, either ES or LPS -induced level of IL-10 in Nrf2 KO macrophages was not as high as those in WT macrophages (Figure 3).

\section{ES -Treated Nrf2 KO Macrophages Failed to Alleviate the Severity of the Colitis in Mice In Vivo}

To evaluate the therapeutic effect of ES -treated macrophage (ES -M) on colitis, we established an acute model of colitis in mice and performed adoptive transfer of these macrophages. We observed that DAI scores of the TNBS group were significantly elevated 3 days after induction (Figure 4A). While adoptive transfer of ES -treated WT macrophages decreased the DAI scores, ES -treated Nrf2 KO macrophage (ES - M (KO)) did not reduce these scores. The weight loss rate of TNBS-induced mice after 3 days was close to $10 \%$. Mice treated with ES-M after TNBS induction showed significant weight loss on day 2 and day 3 (Figure 4B). The TNBS group $(7.617 \pm 0.2927 \mathrm{~cm}$ ) and TNBS + ES -M $(\mathrm{KO})(7.683 \pm 0.2639 \mathrm{~cm})$ group showed colonic shortening, whereas colon length the ES -M group $(9.167 \pm 0.2160 \mathrm{~cm})$ was not shortened compared to TNBS group (Figures 4C, D). In addition, treatment of ES -M, but not ES -M (KO), significantly decreased the level of MPO induced by TNBS (TNBS: $1.499 \pm 0.216$ U/g; ES -M +TNBS: $0.9375 \pm 0.1797$ $\mathrm{U} / \mathrm{g}$; ES $-\mathrm{M}(\mathrm{KO})+\mathrm{TNBS}: 1.465 \pm 0.1248 \mathrm{U} / \mathrm{g}$ ) (Figure 4E).
Histopathological injury in the colon was further measured. We observed that the TNBS group showed distortion of the crypts and extensive cellular infiltration. Administration of ES -M obviously improved the pathological injury, whereas ES -M (KO) failed to alleviate the pathological injury (Figure 4F, G). These results indicated that the treatment of ES -M alleviated the severity of inflammation in the colon. However, no significant difference between TNBS group and TNBS + ES -M (KO) group was evident.

\section{Nrf2 of Macrophages Played a Critical Role in Regulating Cytokine Production Induced by Adoptive Transfer In Vivo}

To assess the induction of Th1 and Th2 immune response, the splenocytes were isolated and assessed by flow cytometry. Compared to the control group, the $\mathrm{CD} 3^{+} \mathrm{CD} 4^{+}$IFN- $\gamma^{+}$T cell population associated with Th1 immune response in TNBSinduced mice was significantly enhanced (Figure 5A). Compared to the TNBS group, Th1 cells in the ES-M instead of ES-M (KO) treatment group were significantly reduced, and the number of $\mathrm{CD}^{+} \mathrm{CD}^{+} \mathrm{IL}-4^{+} \mathrm{T}$ cells was significantly increased, which were defined as Th2 cells (Figure 5A). The ratio (percentage of IL-4/percentage of IFN- $\gamma$ ) in ES -M group $(80.33 \pm 3.327 \%)$ was higher than in the TNBS group (36.03 \pm $1.291 \%)$ and $\mathrm{ES}-\mathrm{M}(\mathrm{KO})$ group $(34.98 \pm 2.263 \%)$ (Figure 5B). IL-10 is essential for the anti-colitis effect (23). Further, we found the population of $\mathrm{CD}^{+} \mathrm{CD}^{+} \mathrm{IL}-10^{+} \mathrm{T}$ cells was increased in ES -M group rather than in ES -M (KO) group (Figure 6).

In addition, cytokine production in the culture supernatant of colon tissue was determined. The level of IFN- $\gamma$ in the colon was significantly elevated in the mice from TNBS group (1178.0 \pm
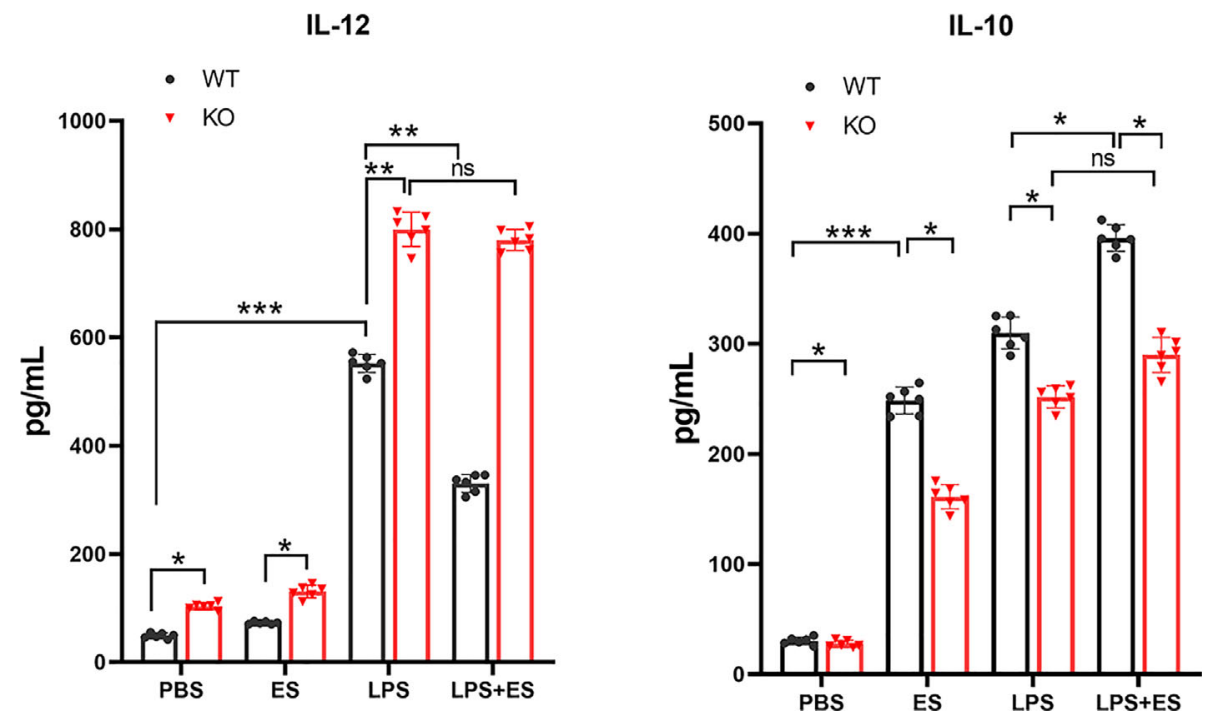

FIGURE 3 | The levels of IL-12 and IL-10 secreted from wild type (WT) or Nrf2 KO macrophages induced by T. spiralis ES. Macrophages were enriched by positive selection with anti- F4/80 magnetic beads. These cells were stimulated with sterile PBS, ES or LPS alone for $24 \mathrm{~h}$. And macrophages were pre-treated with ES $(50 \mu \mathrm{g} / \mathrm{ml})$ for $24 \mathrm{~h}$ before stimulation with LPS $(100 \mathrm{ng} / \mathrm{ml})$ for $24 \mathrm{~h}$. Cell culture supernatants were collected and stored at $-80^{\circ} \mathrm{C}$. IL-12 and IL-10 levels in the supernatant were quantified by ELISA. Results are presented as the mean $\pm \mathrm{SD}(\mathrm{n}=6)$. ${ }^{*} \mathrm{P}<0.05$, ${ }^{\star *} \mathrm{P}<0.01,{ }^{\star \star *} \mathrm{P}<0.001$, $\mathrm{ns}$, no significance, as indicated by line (one-way ANOVA with Tukey's post hoc test). 

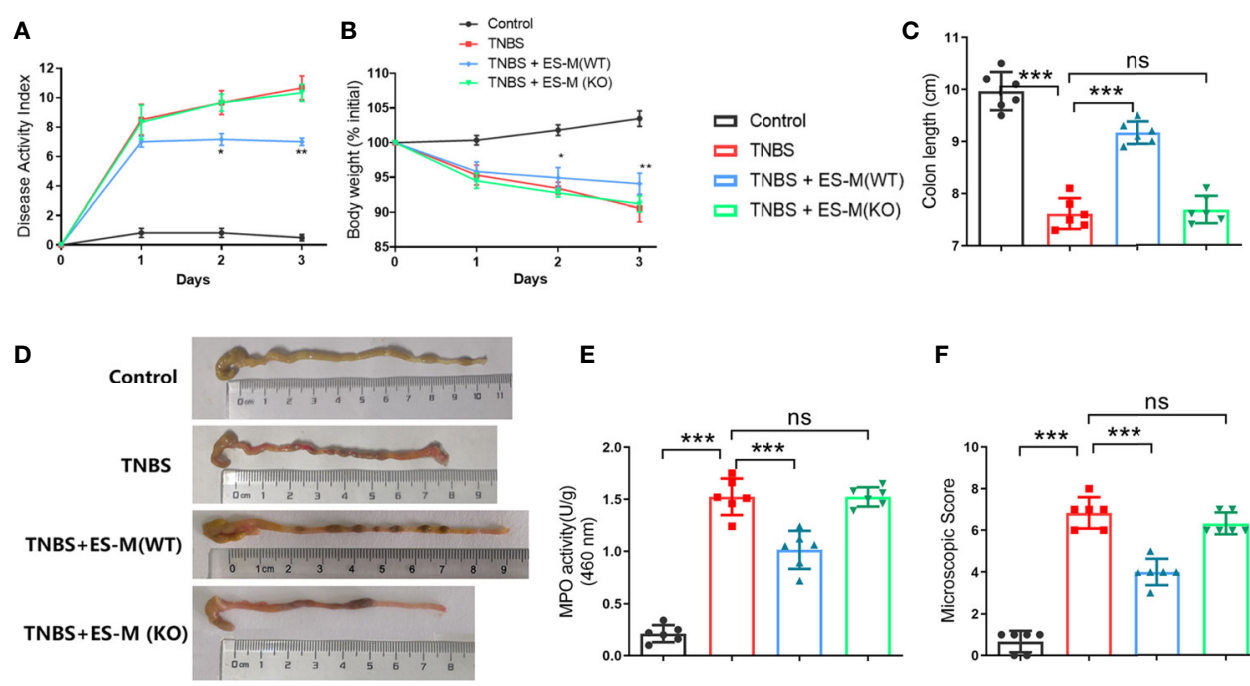

E

$\mathbf{F}$
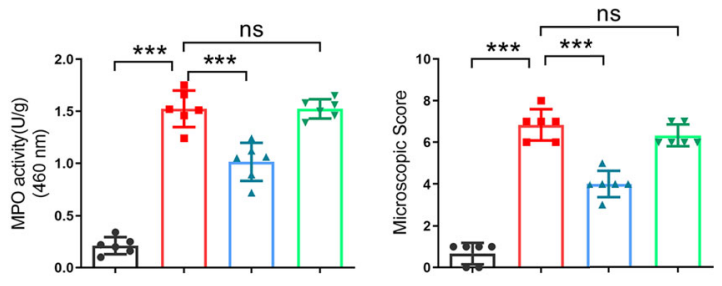

G
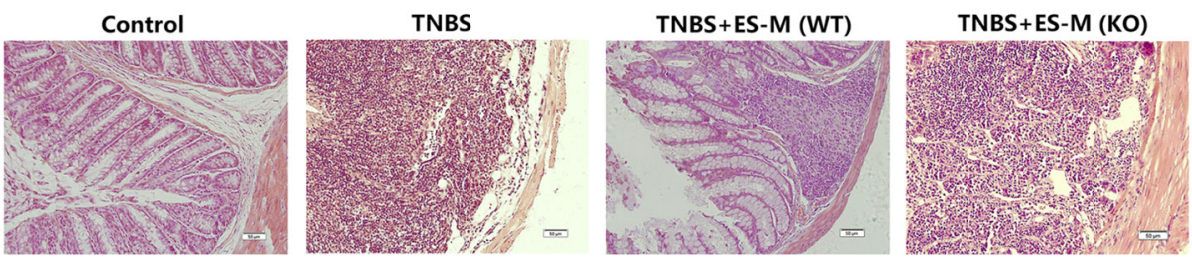

FIGURE 4 | Effect of T. spiralis ES -induced wild type (WT) or Nrf2 KO macrophages (ES-M) on TNBS-induced colitis. For adoptive transfer, WT or Nrf2 KO macrophages induced by $T$. spiralis ES were washed ( $\times 3$ ) with sterile PBS, and $1 \times 10^{6}$ cells in $500 \mu \mathrm{L}$ of sterile PBS were injected intravenously (i.v.) after TNBS challenge. Three days later, colitis was induced by TNBS in these recipient mice. Mice were humanely euthanized by $\mathrm{CO}_{2}$, and then the colon and spleen were collected for the following experiments. The protective efficacy against TNBS challenge was determined in three independent experiments. One representative experiment is shown here. (A) Disease activity index (DAl) was measured during the disease process. (B) The daily mean weight change in each group was calculated. (C, D) After 3 days, colons were removed, and the lengths of their colons were measured and recorded. (E) Myeloperoxidase (MPO) activity in the colonic tissues was detected. The colonic segments were stained with hematoxylin and eosin (H.E.) staining according to standard protocols. (F) The colons from each experimental group ( $n=6$ ) were processed for histological evaluation (200x). (G) Histopathological damage scores were determined for the colon tissue samples. The results are representative of at least three independent experiments and expressed as the mean $\pm S D$ of each group $(n=6) .{ }^{\star \star \star} P<0.001$, ns, no significance, as indicated by line (one-way ANOVA with Tukey's posttest) on the same day.

$82.80 \mathrm{pg} / \mathrm{ml})$ and ES $-\mathrm{M}(\mathrm{KO})+$ TNBS group $(1127.1 \pm 117.79$ $\mathrm{pg} / \mathrm{ml})$, but was significantly inhibited in the ES -M -treated mice $(433.7 \pm 21.35 \mathrm{pg} / \mathrm{ml})$. There was no effect of TNBS alone on IL-4 production $(75.1 \pm 7.51 \mathrm{pg} / \mathrm{ml})$, and ES $-\mathrm{M}$-treated group had significantly higher level of IL-4 (196.3 $\pm 13.02 \mathrm{pg} / \mathrm{ml})$, which is suggested to be associated with the Th2 immune response. ES -M -treated group also exhibited higher level of IL-10 than TNBS group (TNBS + ES -M: $896.3 \pm 56.30$ pg/ml; TNBS: $117.0 \pm 4.858$ $\mathrm{pg} / \mathrm{ml})$. However, treatment of ES -M (KO) did not enhance the levels of IL-4 (74.33 $\pm 10.65 \mathrm{pg} / \mathrm{ml})$ and IL-10 (108.3 $\pm 7.367 \mathrm{pg} /$ $\mathrm{ml}$ ) (Figure 7).

\section{DISCUSSION}

Inflammatory bowel disease (IBD), including the two main clinical entities, Crohn's disease (CD) and ulcerative colitis (UC), are chronic relapsing disorders of the gastrointestinal tract (24). Crohn's disease (CD) is a chronic dysregulated inflammatory disease of intestinal tract. Several studies have demonstrated the therapeutic potential of helminths in the treatment of colitis (2527). Previously, we have demonstrated that infection of Trichinella spiralis reduce the severity of TNBS -induced colitis (28). However, helminth therapy is hard to accept for patients because of many ethical issues that might be raised. In the present study, we aimed to investigate a potentially new approach in the treatment of CD.

Cellular immunotherapy has been emerging as a therapeutic option, and macrophages play central roles in the development of colitis. Helminths and their products promote macrophage differentiation into alternatively activated macrophages (M2) that control the Th1 and upregulate the Th2 immune response (5). It has been reported that helminths protect from colitis through induction of alternatively activated macrophages (M2) (4). Macrophages treated with antigen from helminth can protect against colitis $(9,29,30)$. A recent study (9) has found that adoptive transfer of T. spiralis excretory/secretory (ES) -treated macrophages inhibited dextran sulfate sodium (DSS) -induced UC. However, current evidence suggests that UC is a modified T-helper-2 (Th2) disease, while CD is Th1 driven (31). In this study, we investigated the effect of $T$. spiralis ES -treated 
A

Isotype Control

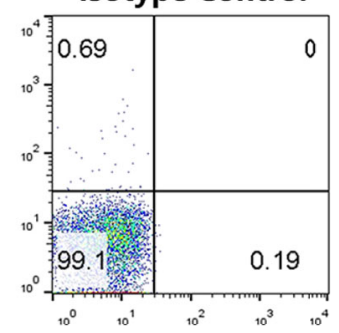

B
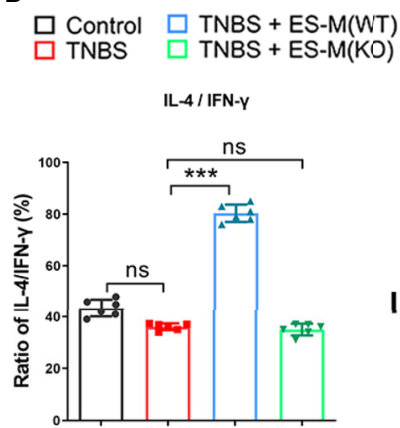

Control

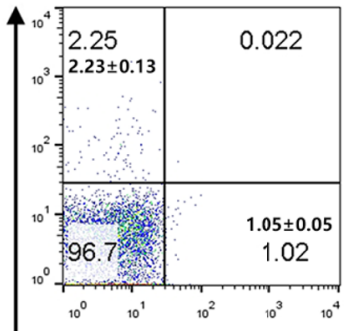

TNBS+ES-M(WT)

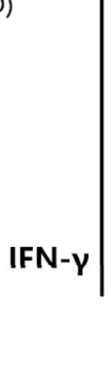

TNBS

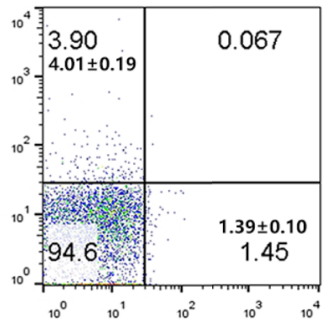

TNBS+ES-M(KO)

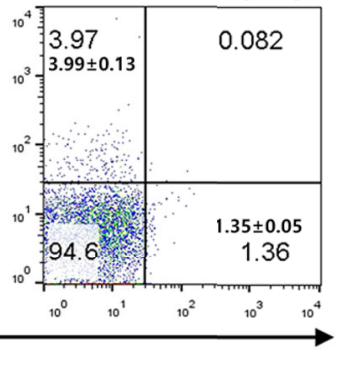

FIGURE 5 | Differentiation of $\mathrm{CD}^{+} \mathrm{CD}^{+}$IFN- $\gamma^{+}$or IL-4 ${ }^{+} \mathrm{T}$ cells of $T$. spiralis ES -induced wild type (WT) or Nrf2 KO macrophages (ES-M) on TNBS-induced colitis. (A) $\mathrm{CD}^{+} \mathrm{CD}^{+} \mathrm{T}$ cells were gated. IFN- $\gamma^{+}, \mathrm{IL}-4^{+} \mathrm{T}$ cells populations were determined. (B) The ratio of IL-4/IFN- $\gamma$ was shown. Data are shown as the means $\pm \mathrm{SD}$ (three independent experiments) of each group $(n=6) .{ }^{* * *} \mathrm{p}<0.001$, $\mathrm{ns}$, no significance, as indicated by line (one-way ANOVA with Tukey's posttest). These figures are representative of three independent experiments.

A

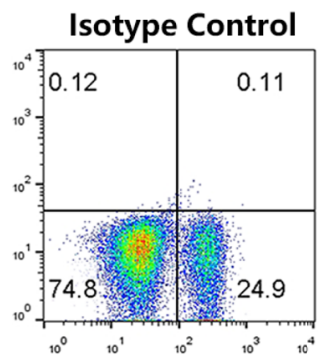

B

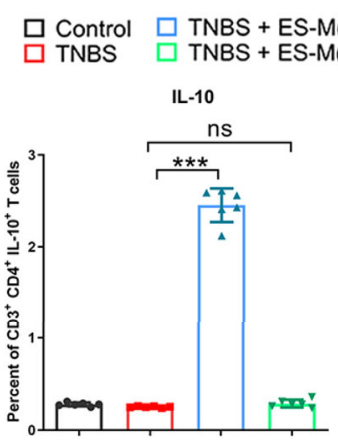

Control

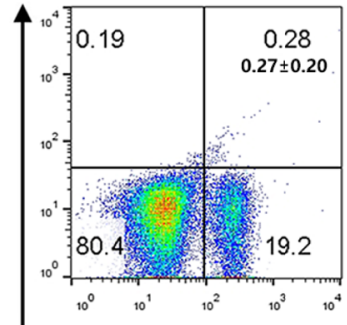

TNBS+ES-M(WT)

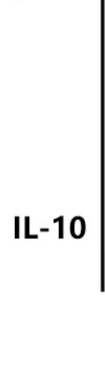

TNBS

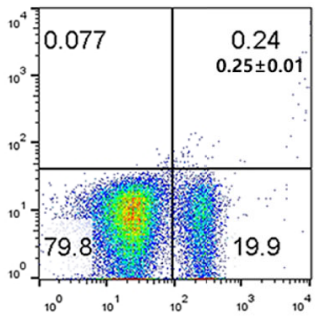

TNBS+ES-M(KO)

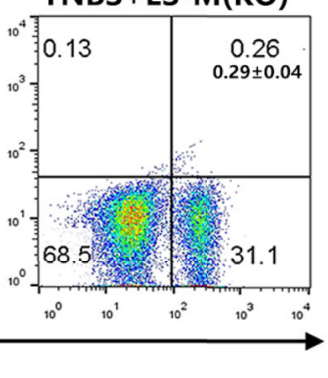

FIGURE 6 | Differentiation of $\mathrm{CD}^{+} \mathrm{CD}^{+} \mathrm{IL}-10^{+} \mathrm{T}$ cells of $\mathrm{T}$. spiralis ES -induced wild type (WT) or Nrf2 KO macrophages (ES-M) on TNBS-induced colitis. (A) CD3 ${ }^{+}$ $T$ cells were gated. $C D 4^{+} \mathrm{IL}-10^{+} \mathrm{T}$ cells populations were determined. (B) The percent of $\mathrm{CD} 4^{+} \mathrm{IL}-10^{+} \mathrm{T}$ cells were shown. Data are shown as the means $\pm \mathrm{SD}$ (three independent experiments) of each group $(n=6) .{ }^{* \star *} \mathrm{p}<0.001$, ns, no significance, as indicated by line (one-way ANOVA with Tukey's posttest). These figures are representative of three independent experiments. 


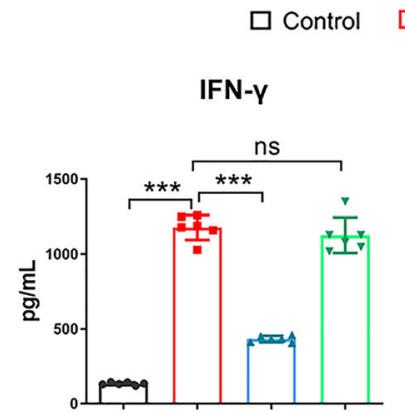

TNBS

$\square T N B S+E S-M(W T)$

IL-4

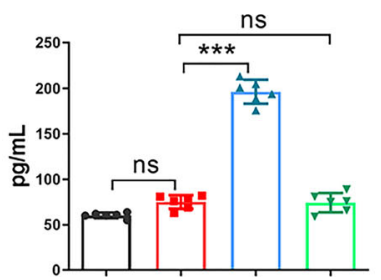

TNBS + ES-M(KO)

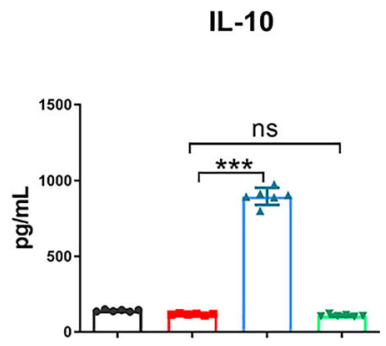

FIGURE 7 | Cytokine production in colons of T. spiralis ES -induced wild type (WT) or Nrf2 KO macrophages (ES-M) on TNBS-induced colitis. The colon culture supernatant was used to determine the cytokine production. IFN- $\gamma$, IL-4 and IL-10 levels were measured by ELISA. Data are shown as the means \pm SD (three independent experiments) of each group $(n=6) .{ }^{\star \star \star} \mathrm{p}<0.001$, ns, no significance, as indicated by line (one-way ANOVA with Tukey's posttest).

macrophage (ES -M) on TNBS -induced colitis, which is a wellestablished model of human CD (32). Our results showed that adoptive transfer of ES -M attenuated the inflammation of TNBS-induced colitis through inhibiting Th1 -related cytokine IFN- $\gamma$. The mice treated with ES -M exhibited increased levels of IL-4 and IL-10 in vivo. IL-4 is produced by Th2 cells and can suppress pro-inflammatory cytokine and alleviate colitis (33). Th2 cytokines can promote the development of goblet cells and mast cells, thereby changing the intestinal environment, especially IL-4 (34). IL-10 has been previously related to Th2 differentiation (35) and is known as an anti-inflammatory cytokines (36). Notably, IL-10 administration have proven efficacy in suppressing colitis in mice (4).

Consistent with this previous study (9), we also found that T. spiralis ES induced M2 in vitro. Macrophages can change their phenotype in response to many different stimuli, a process called activation. The classically activated or inflammatory macrophages (M1) is characterized by the expression of high levels of proinflammatory factor such as inducible nitric oxide synthase (iNOS), CD11c and IL-12 (21). In contrast, M2 function in resolving inflammation and are characterized by the levels of antiinflammatory cytokine IL-10, CD206 and the arginase-1 (Arg-1) (22). We observed that treatment with ES were able to inhibit the increase in M1 markers expression induced by LPS and enhance the M2 markers expression, indicating that T. spiralis ES could shift the phenotype from M1 to M2. However, the mechanism by which T. spiralis ES polarizes macrophages remains elusive.

Notably, increasing colonic inflammation is associated with oxidative stress injury (22). We demonstrated that TNBS -induced mice exhibited high level of MPO activity. Thus, we speculated that T. spiralis ES participate in regulating oxidative stress in macrophages. Nrf2 (nuclear factor E2-related factor 2) has been known for over 10 years, to be the key transcription factor regulating the antioxidant response which is crucial for cytoprotection against extracellular stresses. Nrf2 positively regulates transcription of genes having antioxidant effects (37). Nrf2 expression and activation occurs during the initial contact of some Leishmania species with cells, increasing the amount of gene products associated with M2 macrophage characteristics (38). We also showed that Nrf2 may be involved in altering the phenotype of ES -induced macrophages. Our results are in agreement with a recent study suggesting that $T$. spiralis infection can mitigate inflammation by upregulating Nrf2 expression (16). We found that ES -treated Nrf2 KO macrophages expressed less IL-10, which is essential for the anti- inflammatory effect (23) and a higher level of IL-12 which can promote a Th1 response (39). The capacity of ES to reduce LPS -induced inflammation when lacking Nrf2 was impaired, suggesting that anti-inflammatory role of ES may be primarily involved in the Nrf2 pathway. It has been also reported that $T$. gondii can balance the oxidative stress of IFN- $\gamma$ and TNF- $\alpha$-stimulated macropahges (40). In addition, we not only showed that Nrf2 participated in the development of T. spiralis ES -induced M2, but also verified that Nrf2 KO macrophage induced by $T$. spiralis ES failed to attenuate the severity of colitis and produced lower levels of IL-10 in vivo, suggesting that Nrf2 KO macrophages treated by ES did not exert their antiinflammatory effects on colitis. It has been also reported that Nrf2 results in the production of IL-10 (41).

Moreover, there are many mechanisms that come into play, making the Nrf2 control system very complex. Nrf2 is regulated by microRNAs, including miR-200a (42). It will also be interesting to explore the role of microRNA from T. spiralis in activation of Nrf2. Previously, we provided evidence for the presence of microRNA in different development stages of $T$. spiralis, suggesting a potential regulatory function in the host (43, 44). There are helminth-specific miRNAs in extracellular vesicles (EVs) isolated from $T$. spiralis ES with immunomodulatory potential (20). Prediction of the interactions between miRNAs of Ts-EVs and murine host genes indicates that Nrf2 may be the target regulated by some miRNAs. Further studies are warranted to discovery new miRNA targeting Nrf2. In addition, a protein kinase has recently been shown to have a role in activating Nrf2 (37). We also found several protein kinase families in a previous study (44). Interestingly, many genes encoding superoxide dismutase (SOD), glutathione perxidases and heat shock protein were identified in ML stages of T. spiralis (44), indicating that these antioxidants may play a role in improving antioxidant defenses in stress conditions. Identification of new microRNA or proteins involved in the Nrf2 in macrophages requires further studies. 
Our findings demonstrated that treatment of T. spiralis ES -induced M2 alleviated the severity of TNBS-induced colitis in mice. Furthermore, we confirmed that Nrf2 participated in the development of T. spiralis ES -induced M2 in vitro and played a critical role in effect of ES -M on colitis in vivo. These results provide evidence that $T$. spiralis ES -induced M2 may serve as a potentially new approach for the treatment of CD or other Th1 immune mediated diseases.

\section{DATA AVAILABILITY STATEMENT}

The original contributions presented in the study are included in the article/supplementary materials. Further inquiries can be directed to the corresponding authors.

\section{ETHICS STATEMENT}

All animal experiments were performed according to regulations of the Administration of Affairs Concerning Experimental Animals in China. The protocol was approved by the Institutional Animal Care and Use Committee of Jilin University (20170318).

\section{REFERENCES}

1. Einwachter H. Current Concepts of Pharmacotherapy in Crohn's Disease. Visceral Med (2019) 35:344-7. doi: 10.1159/000504101

2. Banerjee A, Herring CA, Chen B, Kim H, Simmons AJ, Southard-Smith AN, et al. Succinate Produced by Intestinal Microbes Promotes Specification of Tuft Cells to Suppress Ileal Inflammation. Gastroenterology (2020) 159:21012115.e5. doi: 10.1053/j.gastro.2020.08.029

3. Wammes LJ, Mpairwe H, Elliott AM, Yazdanbakhsh M. Helminth Therapy or Elimination: Epidemiological, Immunological, and Clinical Considerations. Lancet Infect Dis (2014) 14:1150-62. doi: 10.1016/S1473-3099(14)70771-6

4. Hunter MM, Wang A, Parhar KS, Johnston MJ, Van Rooijen N, Beck PL, et al. In Vitro-Derived Alternatively Activated Macrophages Reduce Colonic Inflammation in Mice. Gastroenterology (2010) 138:1395-405. doi: 10.1053/ j.gastro.2009.12.041

5. Maizels RM, McSorley HJ. Regulation of the Host Immune System by Helminth Parasites. J Allergy Clin Immunol (2016) 138:666-75. doi: 10.1016/j.jaci.2016.07.007

6. Ledesma-Soto Y, Callejas BE, Terrazas CA, Reyes JL, Espinoza-Jimenez A, Gonzalez MI, et al. Extraintestinal Helminth Infection Limits Pathology and Proinflammatory Cytokine Expression During DSS-Induced Ulcerative Colitis: A Role for Alternatively Activated Macrophages and Prostaglandins. BioMed Res Int (2015) 2015:563425. doi: 10.1155/2015/563425

7. Lopes F, Matisz C, Reyes JL, Jijon H, Al-Darmaki A, Kaplan GG, et al. Helminth Regulation of Immunity: A Three-Pronged Approach to Treat Colitis. Inflammatory Bowel Dis (2016) 22:2499-512. doi: 10.1097/ MIB.0000000000000889

8. Jin X, Yang Y, Bai X, Shi H, Zhang W, Zhang Z, et al. Dendritic Cells Treated by Trichinella Spiralis Muscle Larval Excretory/Secretory Products Alleviate Tnbs-Induced Colitis in Mice. Int Immunopharmacol (2019) 70:378-86. doi: 10.1016/j.intimp.2019.02.028

9. Kang SA, Park MK, Park SK, Choi JH, Lee DI, Song SM, et al. Adoptive Transfer of Trichinella Spiralis-Activated Macrophages can Ameliorate Both Th1- and Th2-Activated Inflammation in Murine Models. Sci Rep (2019) 9:6547. doi: 10.1038/s41598-019-43057-1

10. Steinfelder S, O'Regan NL, Hartmann S. Diplomatic Assistance: Can Helminth-Modulated Macrophages Act as Treatment for Inflammatory Disease? PloS Pathog (2016) 12:e1005480. doi: 10.1371/journal.ppat.1005480

\section{AUTHOR CONTRIBUTIONS}

$\mathrm{XJ}$ and $\mathrm{XB}$ conceived and designed the experiments. $\mathrm{XJ}, \mathrm{XB}, \mathrm{YZ}$, $\mathrm{ZD}$, and JP performed the experiments. XJ, XB, and XL analyzed the data. XJ, XB, and ML drafted the paper. YZ, ZD, JP, XL, and $M L$ revised the paper. All authors contributed to the article and approved the submitted version.

\section{FUNDING}

This study was supported by The National Key Research and Development Program of China (2018YFC1602500, 2017YFC1601200), the National Natural Science Foundation of China $(31520103916,31872467)$ and Program for JLU Science and Technology Innovative Research Team(2017TD-32).

\section{ACKNOWLEDGMENTS}

We thank Xinrui Wang for the technical assistance. Our thanks are also extended to express our gratitude to all the people who made this work.

11. Li J, Wang H, Zheng Z, Luo L, Wang P, Liu K, et al. Mkp-1 Cross-Talks With Nrf2/Ho-1 Pathway Protecting Against Intestinal Inflammation. Free Radical Biol Med (2018) 124:541-9. doi: 10.1016/j.freeradbiomed.2018.07.002

12. Wei HJ, Gupta A, Kao WM, Almudallal O, Letterio JJ, Pareek TK. Nrf2Mediated Metabolic Reprogramming of Tolerogenic Dendritic Cells Is Protective Against Aplastic Anemia. J Autoimmun (2018) 94:33-44. doi: 10.1016/j.jaut.2018.07.005

13. Zhang Y, Yan T, Sun D, Xie C, Wang T, Liu X, et al. Rutaecarpine Inhibits Keap1-Nrf2 Interaction to Activate NRF2 and Ameliorate Dextran Sulfate Sodium-Induced Colitis. Free Radical Biol Med (2020) 148:33-41. doi: 10.1016/j.freeradbiomed.2019.12.012

14. Luo W, Xu Q, Wang Q, Wu H, Hua J. Effect of Modulation of PPAR-Gamma Activity on Kupffer Cells M1/M2 Polarization in the Development of nonAlcoholic Fatty Liver Disease. Sci Rep (2017) 7:44612. doi: 10.1038/srep44612

15. Cho HY, Gladwell W, Wang X, Chorley B, Bell D, Reddy SP, et al. Nrf2Regulated PPAR\{Gamma\} Expression is Critical to Protection Against Acute Lung Injury in Mice. Am J Respir Crit Care Med (2010) 182:170-82. doi: 10.1164/rccm.200907-1047OC

16. Chu KB, Lee HA, Kang HJ, Moon EK, Quan FS. Preliminary Trichinella Spiralis Infection Ameliorates Subsequent Rsv Infection-Induced Inflammatory Response. Cells (2020) 9(5):1314. doi: 10.3390/cells9051314

17. Jin X, Bai X, Yang Y, Ding J, Shi H, Fu B, et al. Nlrp3 Played a Role in Trichinella Spiralis-Triggered Th2 and Regulatory T Cells Response. Veterinary Res (2020) 51:107. doi: 10.1186/s13567-020-00829-2

18. Schwarz H, Schmittner M, Duschl A, Horejs-Hoeck J. Residual Endotoxin Contaminations in Recombinant Proteins Are Sufficient to Activate Human CD1c+ Dendritic Cells. PloS One (2014) 9:e113840. doi: 10.1371/journal.pone.0113840

19. Xu N, Bai X, Liu Y, Yang Y, Tang B, Shi HN, et al. The Anti-Inflammatory Immune Response in Early Trichinella Spiralis Intestinal Infection Depends on Serine Protease Inhibitor-Mediated Alternative Activation of Macrophages. J Immunol (Baltimore Md.: 1950) (2021) 206:963-77. doi: 10.4049/jimmunol.2000290

20. Yang Y, Liu L, Liu X, Zhang Y, Shi H, Jia W, et al. Extracellular Vesicles Derived From Trichinella Spiralis Muscle Larvae Ameliorate TNBS-Induced Colitis in Mice. Front Immunol (2020) 11:1174. doi: 10.3389/fimmu.2020.01174

21. Funes SC, Rios M, Escobar-Vera J, Kalergis AM. Implications of Macrophage Polarization in Autoimmunity. Immunology (2018) 154:186-95. doi: 10.1111/ imm. 12910 
22. Naito Y, Takagi T, Higashimura Y. Heme Oxygenase-1 and AntiInflammatory M2 Macrophages. Arch Biochem biophysics (2014) 564:83-8. doi: 10.1016/j.abb.2014.09.005

23. Narushima S, Spitz DR, Oberley LW, Toyokuni S, Miyata T, Gunnett CA, et al. Evidence for Oxidative Stress in NSAID-Induced Colitis in IL10-/- Mice. Free Radic Biol Med (2003) 34:1153-66. doi: 10.1016/S0891-5849(03)00065-0

24. Axelrad JE, Cadwell KH, Colombel JF, Shah SC. Systematic Review: Gastrointestinal Infection and Incident Inflammatory Bowel Disease. Alimentary Pharmacol Ther (2020) 51:1222-32. doi: 10.1111/apt.15770

25. Driss V, El Nady M, Delbeke M, Rousseaux C, Dubuquoy C, Sarazin A, et al. The Schistosome Glutathione s-Transferase P28GST, a Unique Helminth Protein, Prevents Intestinal Inflammation in Experimental Colitis Through a Th2-Type Response With Mucosal Eosinophils. Mucosal Immunol (2016) 9:322-35. doi: 10.1038/mi.2015.62

26. Eichenberger RM, Ryan S, Jones L, Buitrago G, Polster R, Montes de Oca M, et al. Hookworm Secreted Extracellular Vesicles Interact With Host Cells and Prevent Inducible Colitis in Mice. Front Immunol (2018) 9:850. doi: 10.3389/ fimmu.2018.00850

27. Arai T, Lopes F, Shute A, Wang A, McKay DM. Young Mice Expel the Tapeworm Hymenolepis Diminuta and are Protected From Colitis by Triggering a Memory Response With Worm Antigen. Am J Physiol Gastrointest liver Physiol (2018) 314:G461-70. doi: 10.1152/ajpgi.00295.2017

28. Zhao Y, Liu MY, Wang XL, Liu XL, Yang Y, Zou HB, et al. Modulation of Inflammatory Bowel Disease in a Mouse Model Following Infection With Trichinella Spiralis. Veterinary Parasitol (2013) 194:211-6. doi: 10.1016/ j.vetpar.2013.01.058

29. Ziegler T, Rausch S, Steinfelder S, Klotz C, Hepworth MR, Kühl AA, et al. A Novel Regulatory Macrophage Induced by a Helminth Molecule Instructs Il10 in CD4+ T Cells and Protects Against Mucosal Inflammation. J Immunol (2015) 194:1555-64. doi: 10.4049/jimmunol.1401217

30. Reyes JL, Lopes F, Leung G, Jayme TS, Matisz CE, Shute A, et al. Macrophages Treated With Antigen From the Tapeworm Hymenolepis Diminuta Condition Cd25+ T Cells to Suppress Colitis. FASEB J (2019) 33:5676-89. doi: 10.1096/fj.201802160R

31. Ungaro R, Mehandru S, Allen PB, Peyrin-Biroulet L, Colombel J-F. Ulcerative Colitis. Lancet (London England) (2017) 389:1756-70. doi: 10.1016/S01406736(16)32126-2

32. Camoglio L, te Velde AA, de Boer A, ten Kate FJ, Kopf M, van Deventer SJ. HaptenInduced Colitis Associated With Maintained Th1 and Inflammatory Responses in IFN-Gamma Receptor-Deficient Mice. Eur J Immunol (2000) 30:1486-95. doi: 10.1002/(SICI)1521-4141(200005)30:5<1486::AID-IMMU1486>3.0.CO;2-8

33. Lloyd CM, Snelgrove RJ. Type 2 Immunity: Expanding Our View. Sci Immunol (2018) 3:25. doi: 10.1126/sciimmunol.aat1604

34. Ding J, Bai X, Wang X, Shi H, Cai X, Luo X, et al. Immune Cell Responses and Cytokine Profile in Intestines of Mice Infected With Trichinella Spiralis. Front Microbiol (2017) 8:2069. doi: 10.3389/fmicb.2017.02069
35. Williams JW, Tjota MY, Clay BS, Vander Lugt B, Bandukwala HS, Hrusch CL, et al. Transcription Factor Irf4 Drives Dendritic Cells to Promote Th2 Differentiation. Nat Commun (2013) 4:2990. doi: 10.1038/ncomms3990

36. Hang L, Kumar S, Blum AM, Urban JFJr., Fantini MC, Weinstock JV. Heligmosomoides Polygyrus Bakeri Infection Decreases Smad7 Expression in Intestinal Cd4(+) $\mathrm{T}$ Cells, Which Allows TGF-Beta to Induce Il-10Producing Regulatory $\mathrm{T}$ Cells That Block Colitis. I Immunol (Baltimore Md.: 1950) (2019) 202:2473-81. doi: 10.4049/jimmunol.1801392

37. Pall ML, Levine S. Nrf2, a Master Regulator of Detoxification and Also Antioxidant, Anti-Inflammatory and Other Cytoprotective Mechanisms, is Raised by Health Promoting Factors. Sheng li xue bao: [Acta physiologica Sinica] (2015) 67:1-18.

38. Vivarini AC, Lopes UG. The Potential Role of Nrf2 Signaling in Leishmania Infection Outcomes. Front Cell infection Microbiol (2019) 9:453. doi: 10.3389/ fcimb.2019.00453

39. Piconese S, Barnaba V. Stability of Regulatory T Cells Undermined or Endorsed by Different Type-1 Cytokines. Adv Exp Med Biol (2015) 850:1730. doi: 10.1007/978-3-319-15774-0_2

40. Pang Y, Zhang Z, Chen Y, Cao S, Yang X, Jia H. The Nrf2 Pathway is Required for Intracellular Replication of Toxoplasma Gondii in Activated Macrophages. Parasite Immunol (2019) 41:e12621. doi: 10.1111/pim.12621

41. Sandberg M, Patil J, D’Angelo B, Weber SG, Mallard C. Nrf2-Regulation in Brain Health and Disease: Implication of Cerebral Inflammation. Neuropharmacology (2014) 79:298-306. doi: 10.1016/j.neuropharm.2013.11.004

42. Eades G, Yang M, Yao Y, Zhang Y, Zhou Q. Mir-200a Regulates Nrf2 Activation by Targeting Keap1 Mrna in Breast Cancer Cells. J Biol Chem (2011) 286:40725-33. doi: 10.1074/jbc.M111.275495

43. Liu X, Song Y, Lu H, Tang B, Piao X, Hou N, et al. Transcriptome of Small Regulatory Rnas in the Development of the Zoonotic Parasite Trichinella Spiralis. PloS One (2011) 6:e26448. doi: 10.1371/journal.pone.0026448

44. Liu X, Song Y, Jiang N, Wang J, Tang B, Lu H, et al. Global Gene Expression Analysis of the Zoonotic Parasite Trichinella Spiralis Revealed Novel Genes in Host Parasite Interaction. PloS Neglected Trop Dis (2012) 6:e1794. doi: 10.1371/journal.pntd.0001794

Conflict of Interest: The authors declare that the research was conducted in the absence of any commercial or financial relationships that could be construed as a potential conflict of interest.

Copyright (C) 2021 Jin, Bai, Zhao, Dong, Pang, Liu and Liu. This is an open-access article distributed under the terms of the Creative Commons Attribution License (CC BY). The use, distribution or reproduction in other forums is permitted, provided the original author(s) and the copyright owner(s) are credited and that the original publication in this journal is cited, in accordance with accepted academic practice. No use, distribution or reproduction is permitted which does not comply with these terms. 